\title{
Control de bacteriemia nosocomial pediátrica mediante un programa de cultivo de soluciones parenterales en uso*
}

Juan M. Muñoz, M.C ., M. en C., ${ }^{(1)}$ A lejandro E. Macías, M.C., ${ }^{(1)}$ Francisco J. Guerrero, M.C., ${ }^{(2)}$ Isabel Hernández, M.C., ${ }^{(1)}$ Humberto Medina, Q .F.B., ${ }^{(1)}$ Enrique Vargas, M.C., D r. en C. ${ }^{(1)}$

\begin{abstract}
Muñoz JM, Macías AE, Guerrero FJ, Hernández I, Medina H, Vargas E. Control de bacteriemia nosocomial pediátrica mediante un programa de cultivo de soluciones parenterales en uso. Salud Publica Mex 1999;41 suppl 1:S32-S37.
\end{abstract}

\section{Resumen}

Objetivo. Dado que Klebsiella, Enterobacter y Serratia se multiplican en soluciones parenterales y son responsables de una elevada proporción de bacteriemias en los hospitales de México, se propone una estrategia de control mediante la vigilancia microbio lógica de las soluciones en uso. Material y métodos. Hospital de enseñanza de segundo nivel con 193 camas. A tiende principalmente pacientes de escasos recursos. En 1992 se inició la vigilancia de la esterilidad de las soluciones parenterales en los servicios pediátricos mediante cuatro estrategias: durante la primera etapa se cultivó el total de soluciones en uso. Durante la segunda se cultivaron muestras aleatoriamente elegidas. Tercera y cuarta etapas con muestreo controlado y dirigido, respectivamente. Resultados. Se han cultivado 1940 infusiones. Se ha observado una reducción de la tasa de contaminación (de $29.6 \%$ en 1992 a 12.9\% en 1997, p< 0.001).A simismo se redujo la proporción de bacilos gramnegativos aislados en sangre $(72.7 \%$ vs $40.85 \%, p<0.001)$ y las bacteriemias nosocomiales primarias (BN P) (3.12 vs 1.54 por 100 egresos, $p<0.0001$ ). Conclusiones. La detección de contaminantes señala posibles fallas en el manejo parenteral, áreas de riesgo y pacientes potencialmente afectados. El programa permite estudiar el nivel endémico de contaminación de infusiones y limitar los brotes de bacteriemias nosocomiales primarias a un costo bajo.

Palabras clave: infección hospitalaria; infusiones parenterales; México
Muñoz JM, Macías AE, Guerrero FJ, Hernández I, Medina H, Vargas E. Pediatric nosocomial bacteremia control program based on culturing in use parenteral infusions.

Salud Publica Mex 1999;41 suppl 1:S32-S37.

\begin{abstract}
A bstract
Objective. As Klebsiella, Enterobacter and Serratia are capable of growth in IV fluids and these bacteria are commonly implicated in nosocomial bacteremia, a control strategy through microbiological surveilance of in-use parenteral solutions is proposed. Material and methods. A second level general teaching hospital, serving low-income patients. Through four consecutive strategies, a continous surveillance program of IV fluids sterility in pediatric wards was stablished in 1992. During the first stage all of the in - use solutions were cultured. D uring the second stage randomly selected samples were studied. Third stage was designed as a case-control study.T he last stage included samples drawn in convenience. Positive cultures point out eventual infusion mishandling, as well as high-risk areas and patients. Results. After culturing 1940 parenteral solutions, infusion contamination rates decreased from $29.6 \%$ in 1992 to $12.9 \%$ in $1997(p<0.001)$. The proportion of $\mathrm{G}$ ram-negative rods isolated from blood cultures went from $72.7 \%$ to $40.85 \%$ $(p<0.0001)$, and the nosocomial bacteremia rate dropped from 3.12 to 1.54 per 100 discharges. Conclusions. The program has enabled us to: 1) D etect and control eventual bacteremia outbreaks; 2) Assess the endemic infusion contamination rate; 3) Arouse healthcare workers awareness about infusion line precautions; 4) Have a suitable surveillance strategy according to our laboratory's workload.
\end{abstract}

Key words: cross infection; infusions, parenteral; Mexico

* Resultados parciales presentados en el XXI Congreso Internacional de la Asociación Mexicana de Infectología, Guadalajara, Jalisco, noviembre de 1996.

(1) Facultad de Medicina de León, Guanajuato, México.

(2) Hospital General Regional de León, Guanajuato, México. 
$E$ n general el avance de la medicina se asocia con más procedimientos de invasión al paciente; sin embargo, la falta de vigilancia lo hace sujeto de mayor riesgo. En los países desarrollados se reconoce que la contaminación de los catéteres centrales es responsable de una proporción considerable de bacteriemias nosocomiales primarias (BNP); no obstante, se ha desdeñado el riesgo asociado con la contaminación extrínseca de soluciones parenterales, al considerarla como evento poco frecuente..$^{1-3}$

La bacteriemia nosocomial en pediatría es una de las infecciones nosocomiales (IN) más frecuentes, costosas y letales. En Estados Unidos de América (EUA) se estima una mortalidad atribuible de 16 a $35 \%$, una prolongación de la estancia hospitalaria de 24 días y un incremento en costo hasta de 40000 dólares por paciente. ${ }^{4,5}$ En México, los informes de brotes de BNP pocas veces ofrecen una explicación clara de sus orígenes. ${ }^{6-10}$ Por otra parte, el criterio diagnóstico de bacteriemia está sujeto a la necesidad de efectuar cultivos de sangre y de catéteres en pacientes con sospecha clínica; estos procedimientos no se realizan en muchos hospitales mexicanos, lo que supone un marcado subregistro. Los informes de brotes en México se limitan a enumerar los factores de riesgo que tiene el paciente para desarrollar la enfermedad. Este abordaje sugeriría que el huésped y el agente son importantes, elementos independientes de las conductas del personal. Bajo esta óptica, se ha conceptualizado a los microrganismos como la figura misma de la gravedad del problema. La imagen de bacterias como Enterobacter cloacae, Pseudomonas aeruginosa o Serratia marcescens como productores de enfermedad, parece distraer al clínico de los fenómenos que originan la invasión.

Respecto a los agentes etiológicos de las BNP, en EUA y en Europa se informa del predominio de cocos grampositivos y levaduras. ${ }^{1,11}$ La preponderancia de los bacilos gramnegativos (BGN), en especial los de la tribu Klebsielleae (TK) (Klebsiella, Enterobacter y Serratia) en los brotes mexicanos y en el Hospital General Regional de León (HGRL), sugirió un origen distinto, probablemente derivado de la contaminación de soluciones parenterales, pues estos gérmenes son capaces de multiplicarse en las soluciones con glucosa, que son de uso regular en pacientes pediátricos. ${ }^{1,12-16} \mathrm{El}$ objetivo de este artículo es informar acerca de la experiencia con un programa de vigilancia microbiológica de soluciones parenterales que se inició en 1992 en el HGRL.

\section{Material y métodos}

El HGRL es un hospital de segundo nivel, dependiente de la Secretaría del Estado de Guanajuato, que atien- de a pacientes de bajos recursos. Desde 1992 cuenta con un comité dedicado al estudio y control de las IN, que identificó altas tasas de BNP al analizar los datos de 1990 y 1991. Cuenta también con una enfermera capacitada para la vigilancia epidemiológica y con el apoyo diagnóstico del Laboratorio de Microbiología de la Facultad de Medicina de León. Mensualmente se atienden de 150 a 200 pacientes hospitalizados por más de 72 horas en las salas de pediatría. A partir de 1991 se promovió la toma de hemocultivos de pacientes con sospecha de sepsis, y en 1993 el comité inició la vigilancia de la cloración del agua potable.

\section{Etapas del programa}

Etapa I. Estudio piloto. Cuando se sospechó que la contaminación de soluciones parenterales era la causa del alto nivel endémico de BNP, se efectuó un estudio piloto para el cultivo del total de soluciones parenterales infundidas en septiembre de 1992 en el servicio de pediatría, fuera de un periodo epidémico. ${ }^{15}$

Etapa II. Efecto en las tasas de bacteriemia. Durante esta etapa se cultivaron todo tipo de soluciones parenterales de pacientes con clínica de sepsis o bacteriemia primaria. De no presentarse pacientes febriles, a manera de control se tomaron muestras de un paciente de cada una de las salas. Esta etapa tuvo lugar entre diciembre de 1992 y diciembre de 1993. ${ }^{14}$

Etapa III. Estudio de casos y controles. De julio de 1994 a marzo de 1995 se efectuó un estudio de casos y controles para determinar la fuerza de asociación entre la fiebre o sepsis y la contaminación de infusiones. Se consideró caso a todo paciente que desarrollara fiebre o sepsis nosocomial y se incluyó al paciente más cercano sin patología infecciosa como control. Diariamente se tomaron muestras para cultivo de cualquier solución parenteral en uso de cada uno de los pacientes incluidos.

Etapa IV. Cultivos de escrutinio de soluciones parenterales. Al detectarse un nivel bajo de contaminantes en la etapa III se mantuvo la toma de cultivos de soluciones parenterales a manera de escrutinio. Desde entonces, se toman cultivos de soluciones parenterales a conveniencia, orientados principalmente a pacientes con fiebre o sepsis, considerados de alto riesgo. Ante la aparición de fenómenos epidémicos se intensifica la toma de cultivos y se refuerzan las conductas de cuidados a las líneas de infusión endovenosa. Se integra como parte del programa de control de IN el cultivo por conveniencia de soluciones de nutrición parenteral (NPT) a partir de noviembre de 1995; al menos cuatro muestras son procesadas por mes en el servicio de pediatría. 


\section{Métodos bacteriológicos}

Durante el estudio piloto y la etapa IV el cultivo de soluciones se efectuó mediante la inoculación de 0.5 $\mathrm{ml}$ de la solución parenteral, obtenida del puerto de aplicación de medicamentos, en caldo BHI (brain-heart infusion) (cultivo cualitativo) y $0.1 \mathrm{ml}$ en placa de agar sangre con el método de dispersión (cultivo cuantitativo). La incubación se efectuó a $35^{\circ} \mathrm{C}$ durante 18 a 48 horas; los caldos turbios se resembraron en placa y los gérmenes aislados fueron identificados mediante procedimientos convencionales. En las etapas II y III sólo se efectuaron cultivos cualitativos, de acuerdo con las experiencias del estudio piloto, en el que se encontró crecimiento masivo de manera consistente cuando el caldo enturbió. Los aislamientos de bacterias del género Bacillus fueron considerados contaminantes relacionados con el procedimiento y excluidos del análisis. Los cultivos de sangre se efectuaron de acuerdo con procedimientos previamente descritos y dirigidos sólo a pacientes con fiebre o sepsis. ${ }^{17}$

Se informó a los médicos tratantes acerca de la identidad y el patrón de resistencia de los gérmenes aislados y la implicación potencial, para el retiro inmediato de los sistemas contaminados. Las decisiones de manejo quedaron a cargo de los médicos tratantes.

\section{A nálisis estadístico}

La comparación de proporciones de cultivos positivos del inicio y fin de este informe fueron sometidas a prueba de $c^{2}$, y se muestran los valores de $p$ para cada comparación, así como los intervalos de confianza. Las tendencias y proyecciones de las tasas de IN se estimaron mediante el programa computadorizado NCSS. $^{18}$

\section{Resultados}

En el estudio piloto se cultivaron 230 soluciones, de las cuales 68 (29.5\%) estaban contaminadas. Se encontró un predominio de BGN de la TK $(51 / 68,92.8 \%)$. La tasa de bacteriemia estimada para este periodo resultó en $4.3 \% .{ }^{15}$ Durante la etapa II se cultivaron 1277 infusiones con una tasa de contaminación de $6.8 \%$ $(87 / 1277) \cdot{ }^{14}$ En la etapa III se cultivaron 371 muestras provenientes del seguimiento de 208 pacientes (casos vs controles 1:1) con estancia promedio de 2.8 días. Sólo se encontraron tres contaminaciones $(0.8 \%)$, por lo que no fue posible definir asociaciones sólidas entre los fenómenos de fiebre o sepsis frente a la contaminación de soluciones parenterales. En la etapa IV se han cultivado 62 soluciones parenterales y 239 soluciones de NPT (hasta diciembre de 1996). La tasa de contaminación de las soluciones parenterales fue de $12.9 \%$ $(8 / 62)$, y para las NPT resultó en $7.53 \%(18 / 239)$. De los gérmenes aislados, un tercio $(6 / 18)$ correspondió a BGN con predominio de Pseudomonas spp. (3/6), seguido de Enterobacter spp. (2/6). En el cuadro I se sintetizan la metodología y los resultados de las distintas etapas.

\section{Bacteriología y tendencias de cultivos}

Se cultivaron un total de 1940 soluciones parenterales. De noviembre de 1995 a diciembre de 1996, la tasa de positividad fue de $12.9 \%$ (8/62), resultados significativamente diferentes a los obtenidos durante el estudio piloto $\left(c^{2}=4.17, p=0.041\right.$, RM 3.25); todos los aislamientos efectuados en 1996 han mostrado contaminación masiva.

Los gérmenes aislados de 135 hemocultivos anteriores al programa de control de BNP (agosto de 1990

\section{Cuadro I \\ Diseño y Resultados globales del PROgRa MA de CULtivo de soluciones Parenterales}

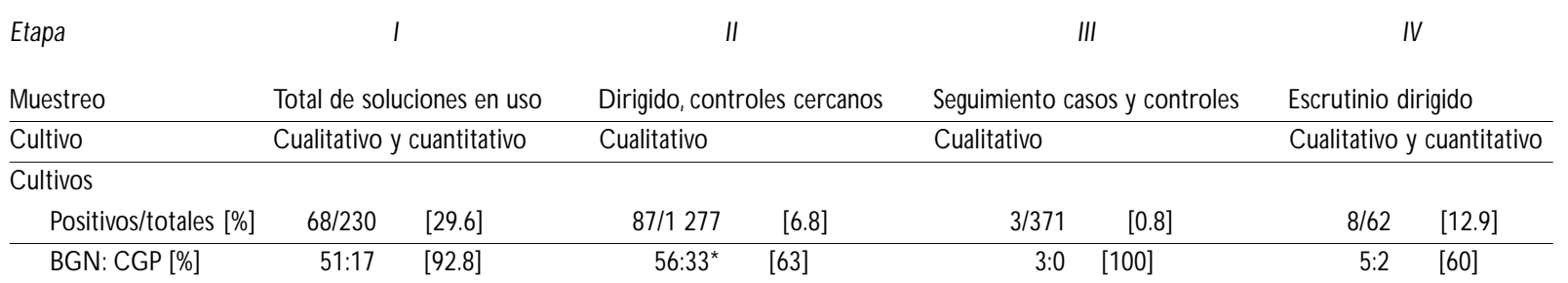

CGP: cocos grampositivos

$B G N$ : bacilos gramnegativos

* Dos contaminaciones mixtas 
a septiembre de 1992) se muestran en la figura 1 comparados con los aislamientos de 71 hemocultivos en 1996. Se observó un descenso en la proporción de BGN $\left(c^{2}=36.29, p<0.001\right)$.

\section{Evolución de la tasa de bacteriemia nosocomial}

La tasa de BNP ha disminuido de 3.12 episodios por 100 egresos en el periodo marzo-junio de 1992, a 1.54 en julio-diciembre de 1996 ( $\mathrm{c}^{2}$ para tendencias $=2028.5$ $p$ - 0.0001). Se observó un comportamiento endémico, con picos estacionales hacia los últimos cuatro meses de cada año, que paulatinamente tienden a ser de menor magnitud; la disminución fue más notoria entre la primera y segunda etapa del estudio, aunque la tendencia es discretamente negativa, aun despreciando el primer periodo como puede verse en la figura 2. La proyección estimada para el nivel endémico de las BNP para el fin de 1999, se calculó en 0.63 bacteriemias por 100 egresos (error estándar 95\%=1.25). En comparación, la tasa global del resto de las IN no ha mostrado un descenso (figura 3).

\section{Discusión}

Los gérmenes aislados en los hospitales de países desarrollados sugieren que los orígenes de las BNP están relacionados con la contaminación de catéteres vasculares. ${ }^{1}$ Este mecanismo no parece ser la fuente principal en los hospitales mexicanos, toda vez que la bacteriología es diferente. De ser así, el abordaje pro-

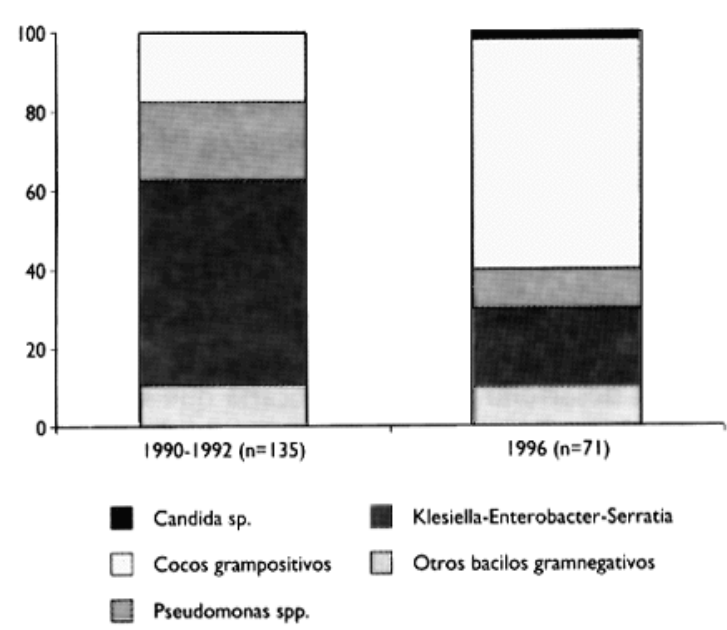

Figura 1. Gérmenes ais.ados en hemocultivos, 19901992 vs1996. Méxıco, 1996

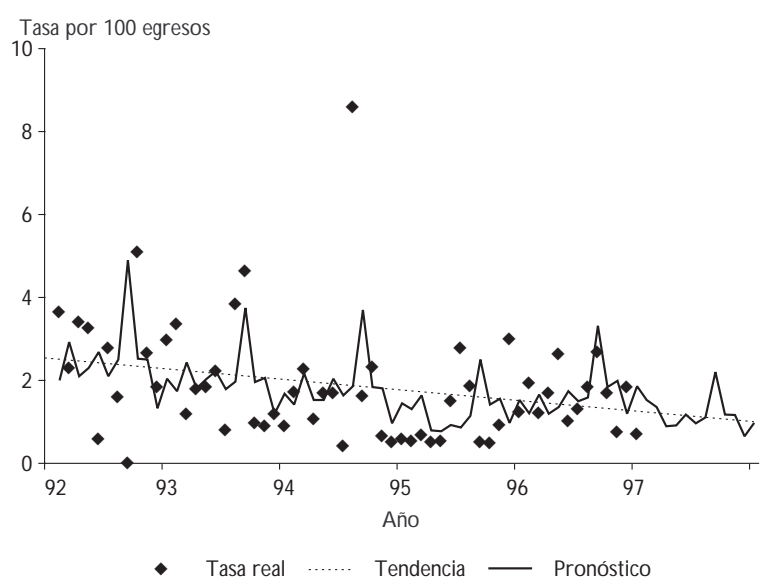

$\mathrm{r}=-0.267$, error estándar del pronóstico $=1.21$

1992-93: tasa 2.57 , IC $95 \%=1.81-3.33$

1996-97: tasa 1.73 , IC $95 \%=1.23-2.23$

Figura 2. Tasas, tendencias y proyección de bacteriemia nosocomial pediátrica. México, 19921997

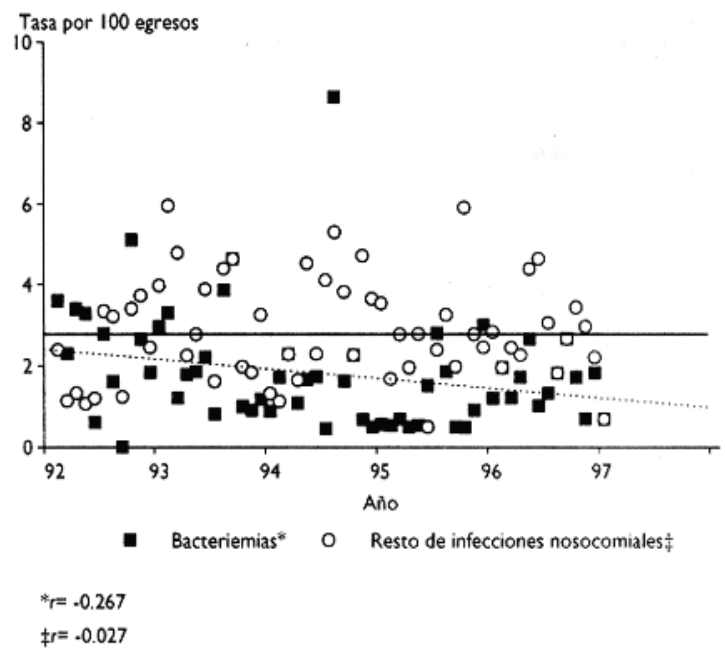

Figura 3. TAsasy tendencias de bacteriemias frente AL Resto de INFECCIONES NOSOCOMIALES, MéXICO, 19921997

puesto para el control de BNP ha de ser distinto al propuesto en EUA. ${ }^{19}$

El descubrimiento, en 1992, de que un tercio de las soluciones parenterales infundidas estaban contaminadas masivamente por BGN, era contrario a la idea que se tenía acerca de la inocuidad de las soluciones parenterales comunes. Este hecho contrastaba con 
las 16 contaminaciones notificadas en 1374 (1.16\% soluciones), ${ }^{1}$ lo que generó serios cuestionamientos acerca del origen de las BNP en el HGRL. Una vez que se eliminó de manera razonable la posibilidad de contaminación intrínseca (del fabricante) como explicación, se propuso que la práctica de conductas de riesgo (compartir jeringas, preparar soluciones sin condiciones de esterilidad) llevan los BGN de paciente a paciente $y$, eventualmente, generan niveles endémicos y brotes de BNP. ${ }^{15}$ Probar una clara relación de causaefecto requería, entonces, evidenciar asociaciones significativas entre contaminantes de soluciones y sepsis o bacteriemia. Tal asociación no se ha establecido, toda vez que detectar contaminación en las soluciones exige su retiro inmediato, abortando probablemente la evolución de la enfermedad. La tolerancia a las soluciones contaminadas podrá ser variable y depender de la madurez del sistema inmune, la intensidad del inóculo infundido, el tiempo de administrado y el microrganismo.

Establecer una relación clara dependerá en gran medida de la capacidad diagnóstica del ambiente en el que se da el fenómeno; por ello, se considera razonable aceptar el diagnóstico tentativo de bacteriemia ante el hallazgo de soluciones parenterales masivamente contaminadas.

En el HGRL existe un descenso en las tasas de BNP. La tendencia observada para el resto de las IN es diferente; de hecho, aparentan mantener tasas estables o incluso ascendentes probablemente por efecto de la búsqueda (figura 3). Aun así, son preocupantes las tendencias recientes al incremento en las BNP. Los cambios podrían estar relacionados con las líneas de NPT adicionadas en 1995 al manejo de los pacientes pediátricos.

En México ya se ha descrito que la mezcla casera de soluciones parenterales es un marcador de riesgo para adquirir BNP en pediatría, ${ }^{8}$ por lo que también se supone esa práctica, entre otras, como fuente de contaminación de soluciones. Mecanismos similares parecen explicar informes de BNP en los que se sugiere "un mecanismo vertical de transmisión" y "fuente común no identificada" de cepas de Klebsiella pneumoniae en Guadalajara y la Ciudad de México, respectivamente. ${ }^{9,10}$ De manera paralela, conviene señalar informes de BNP y paludismo nosocomial propagados por compartir jeringas o medicamentos contaminados. ${ }^{20,21}$

Durante la primera y la cuarta etapas del programa, hubo una disminución significativa de la tasa de contaminación de soluciones. Las estrategias de culti- vo fueron variadas y las tasas de contaminación pudieran no ser equiparables. Si bien se tiene evidencia de la utilidad del cultivo de escrutinio de soluciones, aún queda por definir la mejor estrategia a seguir, para lo cual es necesaria la experiencia de otros grupos de trabajo. Los resultados de la etapa III generaron cierta complacencia y se observaron nuevas elevaciones en la tasa de BNP, lo que sugeriría que es de mayor utilidad la vigilancia transversal periódica dirigida a los casos problema. Además, podría efectuarse un programa de control aleatorio.

Es factible que muchos hospitales mexicanos compartan las limitaciones que aquí se exponen y, con ello, los riesgos de contaminar las soluciones. Poco se ha escrito en México acerca de las tasas de BNP y menos aún de los niveles endémicos de contaminación de soluciones. Si esto es verdad, resultaría grave desatender la contaminación de soluciones parenterales como posible origen de las BNP.

La definición de BNP limita la evaluación, pues muchos hospitales del país carecen de servicios de microbiología clínica. Así, muchos pacientes pudieran enfermar de manera inadvertida con BNP. Si bien la definición de bacteriemia relacionada con la infusión de soluciones parenterales (infusate-related bloodstream infection) requiere cultivos positivos con el mismo germen de la sangre y solución infundida, ${ }^{19}$ resultaría poco razonable complicar aún más el diagnóstico. Se considera que la sola contaminación masiva de la infusión es un criterio diagnóstico suficiente de BNP para los fines de la epidemiología de hospitales semejantes al HGRL.

La cateterización endovenosa ha caído en una falsa impresión de inocuidad y, probablemente, la contaminación de las soluciones durante su uso es la que genera una elevada proporción de las BNP.

A menudo se atribuye una elevada letalidad y contagiosidad al germen mismo; señalar a Serratia, Klebsiella o Enterobacter como responsables sugiere serios problemas durante los brotes epidémicos. Se confiere entonces la peligrosidad del fenómeno a la identidad del germen y no al ambiente que introdujo la enfermedad. La búsqueda de factores de riesgo en los pacientes para el desarrollo de BNP implicaría que se trata de una enfermedad de causalidad múltiple. Se transfiere entonces la responsabilidad de los trabajadores y del ambiente nosocomial al paciente. Sin embargo, cada médico, hospital o sala someterá a los pacientes a un riesgo de magnitud diferente, condición susceptible de ser modificada. Son los hospitales los responsables de exponer-o dejar de hacerlo- a cada paciente al ries- 
go de sepsis -en función de la calidad de los cuidados médicos- $\mathrm{y}$, en menor grado, la susceptibilidad de los pacientes.

Los costos de este programa de vigilancia bacteriológica de las soluciones parenterales han sido menores a aquellos que se hubieran generado por la atención a los pacientes sépticos. La reducción hasta ahora alcanzada de 1.19 BNP por 100 egresos disminuiría aproximadamente 15.43 episodios por semestre, lo que significa de acuerdo con cálculos conservadores, una reducción de 150 días de estancia, tres defunciones y 450000 pesos. ${ }^{5,22}$

Con base en los resultados, se considera útil modificar conductas del personal médico y de enfermería. Quienes indican soluciones parenterales, han de limitar el uso de mezclas efectuadas en los servicios, así como el tiempo de permanencia y número de accesos vasculares. El personal de enfermería deberá estar adiestrado para evitar compartir jeringas y soluciones para mezclas o diluciones de medicamentos. El comité de IN deberá ver en cada BNP por BGN una infección de alta contagiosidad, probablemente generada y propagada por medio de la contaminación de soluciones parenterales. La investigación y la vigilancia de la esterilidad de las soluciones en uso puede contribuir sensiblemente al cuidado de los pacientes pediátricos, sobre todo en hospitales con elevadas tasas de mortalidad nosocomial o con brotes de BNP por BGN. Muchos cuestionamientos podrían surgir respecto del comportamiento de cada especie o cepa bacteriana; sin embargo, parece más razonable atender y mejorar las prácticas hospitalarias de manejo de soluciones y líneas endovenosas a fin de que se limite la contaminación de las líneas de infusión.

La instalación de campanas de flujo laminar o centrales de preparación de soluciones y medicamentos podrá ser uno de los objetivos de los hospitales, pero la adquisición de una conciencia de cuidados en la preparación y el manejo de las soluciones de infusión es más factible, económica y cercana a nuestras posibilidades.

\section{Referencias}

1. Goldmann DA, Pier GB. Pathogenesis of infections related to intravascular catheterization. Clin Microbiol Rev 1993;6:176-192.

2. Snydman DR, Reidy MD, Perry LK, Martin W J. Safety of changing intravenous (IV) administration sets containing burettes at longer than 48 hours intervals. Infect Control 1987:8:113-116.
3. Maki DG , Botticelli JT, LeRoy ML,Thielke TS. Prospective study of replacing administration sets for intravenous therapy at 48- vs 72 - hour intervals. JAMA 1987;258:1777-1781.

4. Centers for D isease Control. Report of the nosocomial infections surveillance system: N osocomial infection rates of interhospital comparison. Infect Control Hosp Epidemiol 1991;12:609-616.

5.JarvisW R. Selected aspects of the socioeconomic impact of nosocomial infections: Morbidity, mortality, cost, and prevention. Infect Control Hosp Epidemiol 1996;17:552-557.

6. N avarro-Montagner FJ, Echavarría-Ybargüengoitia JL,Yáñez-C ontreras ML, Espinoza-Luna R, Ruelas-O rtega P. Bacteriología y factores de riesgo de septicemia en una unidad de cuidado intensivo neonatal. Bol Med Hosp Infant Mex 1987;44:735-738.

7. Flores-Calderón J, Escobedo-Chávez E, Franco del Río G, 0 rtega-Guzmán S, Lavalle-Villalobos A, Moncada-Barrón D. Brote por Serrratia marcescens en un servicio de neonatología. Bol Med Hosp Infant Mex 1988;45:512-516.

8. Zaidi-Jacobson M, Sifuentes J, Bobadilla M, Moncada D, Ponce de León RS. Epidemic of Serratia marcescens bacteremia and meningitis in a neonatal unit in Mexico City. Infect Control Hosp Epidemiol 1989;10:14-20.

9. Pérez-Miravete A. Hemocultivos: experiencia en el Hospital Infantil de México (1990-91). Enferm Infecc Microbiol 1992;12:188-191.

10. G aytán-Meza JJ, Mancilla-Ramírez J,A r redondo-G arcía JL,Alcalá-Padilla L, Ramírez-Valdivia JM, Anthor-Rendón J et al. Etiología de sepsis neonatal y sensibilidad a los antibióticos en el nuevo Hospital Civil de Guadalajara. Enferm Infecc Microbiol 1996;16:80-85.

11. Banerjee SN , Emori TG, C ulver DH, Gaynes RP, Jarvis W R, Horan T et al. Secular trends in nosocomial primary bloodstream infections in the United States, 1980-1989. Am J Med 1991;91(suppl 3B):86S-89S.

12. Mackel DC, Maki D G,Anderson RL, Rhame FS, Bennett JV. N ationwide epidemic of septicemia caused by contaminated intravenous product: Mechanisms of intrinsic contamination. J Clin Micribol 1975;2:486-497.

13. Maki DG, Goldman DA, Rhame FS. Infection control in infusion therapy.Ann Intern Med 1973;79:867-887.

14. Macías-Hernández AE, Hernández-Ramos I, Muñoz-Barret JM,VargasSalado E, Guerrero-Martínez FJ, Medina-Valdovinos $\mathrm{H}$ et al. Pediatric primary gram-negative bacteremia: A possible relationship with infusate contamination. Infect Control Hosp Epidemiol 1996;17:276-280.

15. Macías-Hernández AE, 0 rtega-G onzález P, Muñoz-Barret JM, Hernández-Ramos I, Cal y Mayor I, Guerrero-Martínez FJ et al. Pediatric nosocomial bacteremia. Culturing infusion liquids may help in its control. Rev Invest Clin 1994;46:295-300.

16. Maki DG, Martin W T. N ationwide epidemic of septicemia caused by contaminated infusion products. IV. G rowth of microbial pathogens in fluids for intravenous infusion. J Infect D is 1975;131:267-272.

17. Bartlet RC, Ellner PD, W ashington II JA, Cumitech I. Blood cultures. Washington, D.C.:A merican Society for Microbiology, 1974.

18. N umber C runcher Statistical System (N C SS).Version 6.02.21 Hintze J. 1986.

19. Sherertz RJ. Surveillance for infections associated with vascular catheters. Infect Control Hosp Epidemiol 1996;17:746-752.

20.A bulrahi HA, Bohlega EA, Fontaine RE,Al-Ruwais AA. Plasmodium falciparum malaria transmitted in a hospital through heparin locks. Lancet 1997;349:23-25.

21. Bennett SN, MCN eil MM, Bland LA, A rduino MJ,Villarino ME, Perrotta $D M$ et al. Postoperative infections traced to contamination of an intravenous anesthetic, propofol. N Engl J Med 1995;333:147-154.

22.Vaqué J, Rosselló J,Trilla A, MongeV, G arcía-C aballero J,A rribas JL et al. N osocomial infections in Spain: Results of five nationwide serial prevalence surveys (EPIN E project, 1990 to 1994). Infect Control Hosp Epidemiol 1996;17:293-297. 